\title{
In-vitro anticandidial efficacy of tick egg wax from Hyalomma marginatum, Rhipicephalus bursa and Dermacentor marginatus
}

\author{
Nazli Bilgin ${ }^{1}$ (D), Mayram Hacioglu² (D), Cagla Bozkurt Guzel ${ }^{2}$ (D) Berna Erdal $^{1}$ (D), Sirri Kar ${ }^{3,4}$ (D) \\ ${ }^{1}$ Tekirdag Namik Kemal University, School of Medicine, Department of Medical Microbiology, Tekirdag, Turkey. \\ ${ }^{2}$ Istanbul University, Faculty of Pharmacy, Department of Pharmaceutical Microbiology, Istanbul, Turkey. \\ ${ }^{3}$ Tekirdag Namik Kemal University, Department of Biology, Tekirdag, Turkey. \\ ${ }^{4}$ University of Texas Medical Branch, Department of Microbiology and Immunology, Galveston National Laboratory, Galveston, TX, USA. \\ Correspondence Author: Sirri Kar \\ E-mail: sirrikar@yahoo.com
}

Received: 23.02.2020 Accepted: 12.03 .2020

\begin{abstract}
Objective: In the previous studies, the antibacterial, antifungal, and antiviral efficacy of the tick egg wax-coating of certain tick species were examined and some significant results were obtained. However, related researches and studied tick species are limited. There are hundreds of tick species, and it is well known that the antimicrobial efficacy of the wax is closely related to the species. The aim of this study was to investigate the in-vitro anticandidial efficacy of the egg waxes belonging to three tick species, which have not been studied before and have quite different biological and ecological differences.

Methods: In the study, the egg waxes of the tick species, Hyalomma marginatum, Rhipicephalus bursa, and Dermacentor marginatus, were used on Candida albicans ATCC10231, Candida parapsilosis ATCC 22019, and Candida tropicalis ATCC 750. Antimycotic susceptibility test was carried out in accordance with the Clinical and Laboratory Standards Institute (CLSI) recommendations using the M27-A3 microdilution method. Results: It was determined that the wax of Rhipicephalus bursa has inhibitory effect on Candida tropicalis ATCC 750 in a particular concentration, and no significant effects were observed in other trials.

Conclusion: Anticandidial effect obtained from the egg wax of $R$. bursa can be associated with some distinctive biological characteristics, and it was concluded that the detailed studies with different tick species might yield significant results for the discovery of new generation antifungals. Keywords: Antifungals, tick egg wax, Rhipicephalus bursa, Candida
\end{abstract}

\section{INTRODUCTION}

It has been reported that there are about 20 Candida species among the clinically important fungi seen worldwide, and of those, Candida albicans, the most common fungal pathogen in humans, has been reported to cause 250,000-400,000 deaths annually (1). Both cutaneous and systemic forms of candidiasis can be encountered, and the mortality can reach up to $40 \%$ in systemic $C$. albicans infections (2). Although $C$. albicans is the main agent of candidiasis, other pathogenic species such as C. glabrata, C. parapsilosis, C. tropicalis, C. krusei, and C. lusitaniae (non-albicans candida/ NAC) are frequently isolated from the cases. Furthermore, NAC species are more common in some regions in the world, and a steady increase has been observed in NAC-related cases in the last decades (e.g., 2-10 times in the cases of systemic candidiasis). This increase in the prevalence has been reported to be associated with high antifungal resistance (e.g., specific azole resistance in C. glabrata and C. krusei) seen in this group, and this problem has been indicated to be exacerbated by the irregular antifungal use (3-5). In C. albicans and NAC species, as well as many other fungi, significant antimycotic resistance, which has intrinsic or microbiological character, has been well documented $(6,7)$. For example, single or multiple resistances in various Candida species against different antifungals, such as fluconazole, voriconazole, amphotericin $B$, and caspofungin, have been reported from different parts of the world (6-10). The antifungal diversity in the clinical use is reported to be relatively low compared to the causative agent and disease variety in fungal infections. The widespread, repetitive, and irregular use of antifungal drugs are possibly the main reasons of the resistances. It was anticipated that this situation requires the discovery of new drugs, and moreover, this need will increase over time (6-7). At this point, due to the problems in the medical area such as the resistances and side-effects of the current chemical therapies, the researches on natural substances, which may ensure to overcome these problems, have particular 
importance. The current status of the researches in this area is expressed as "a New Golden Age of natural products drug discovery is dawning" (11). On the other hand, most of the related studies are at the beginning level. The applications are mostly at the stage of using total extracts or selected fractions and the results are given as effective or noneffective $(12,13)$.

In order to determine the natural alternatives that can be used in the treatment of fungal infections, various substances from different origins have been investigated such as microorganismal substances (14), sea sponge (15), the poison of honey bee (Apis mellifera) (16), the larval secrets of a lepidopteran parasitoid, Pimpla turionellae L. (Hymenoptera: Ichneumonidae) (17), and the total isolates or some specific fractions of many kind of plant species (13, 18).

Tick egg wax is one of the sources investigated in the process of determining natural antimycotics. In ixodid ticks, the biological process includes eggs, larvae, nymphs, and adults (male and female). After detachment from the host, engorged adult female ticks hide in a suitable area on the ground and then they deposit up to thousands of eggs in a period that can last for several days or weeks before they die. The eggs, each 50-100 $\mu \mathrm{g}$ in weight and 0.5-1 mm, are laid as a batch. The surfaces of the eggs were covered with the wax, which consists of the secretions of the epithelial cells and accessory glands of the reproductive tract, the porose area, and principally the Gené organ which is a femalespecific gland $(19,20)$. It has been reported that the wax, which thickness varies between 0.5-2.0 $\mu \mathrm{m}$ (21), has an average amount of $24 \mathrm{mg}$ per gram eggs mainly according to the tick species (22). The wax is known to have some vital missions for the eggs such as holding the egg mass together, protecting the eggs against drying, environmental physical, chemical or microbiological factors, and ensuring the proper gas exchange between eggs and air $(20,21,23,24)$.

In the previous studies, the egg waxes of Amblyomma habreum $(25,26)$ A. cajennense (27-29), A. aureolatum (29), Boophilus (Rhipicephalus) microplus (29-32), Rhipicephalus sanguineus (29), Haemaphysalis longicornis, H. doenitzi, Dermacentor silvarum and Hyalomma asiaticum (22) have been tested against various bacteria, fungi, and viruses, and some considerable results were obtained. However, these studies have also revealed that the antimicrobial efficacy of the wax varies dramatically related to the tick species. In this study, the egg waxes of three tick species ( $H$. marginatum, $R$. bursa, and D. marginatus), which have not been studied before and have quite different biological and ecological features, were investigated in terms of in-vitro anticandidial efficacy on three Candida species.

\section{METHODS}

\subsection{Tick eggs}

Non-infected laboratory colonies of $H$. marginatum, $D$. marginatus and $R$. bursa were used in the study. The maintenance of the colonies was carried out at Tekirdag Namik Kemal University Experimental Animal Application and Research Center, within the framework of the permissions received from the Tekirdag Namik Kemal University Animal Experiments Local Ethics Committee (2014/08-04). New Zealand rabbits (Oryctolagus cuniculus) were used to feed all of the developmental stages (larvae, nymphs, and adults). After the detachment from the host, the engorged females were washed with distilled water, dried, taken into sterile tubes individually and placed in the incubator. The conditions of the incubator were set at $25-27{ }^{\circ} \mathrm{C}$ and $70-80 \%$ humidity for $\mathrm{H}$. marginatum and R. bursa, and at $20-22{ }^{\circ} \mathrm{C}$ and $80-90 \%$ humidity for $D$. marginatus. The process of the egg laying was regularly monitored, and the eggs were examined under a stereomicroscope. Incubation was continued until the first laid eggs reached the advanced stages (just before the larva began to hatch) of embryogenesis. Thus the wax extract was intended to include some possible changes which can occur in the egg wax during and after the laying, e.g., hardening in the texture, increase in the adhesive properties, and reduction in the levels of unsaturated fatty acids caused by oxidation $(21,23,33)$. After this incubation egg batches were taken from the tubes and weighed, taken into sterile tubes and kept at $-80^{\circ} \mathrm{C}$ until the extraction of the wax.

\subsection{Extraction of the tick egg wax}

In the wax extraction process, previously described method was used (25). The following procedure has been carried out to include all the fractions of the wax to the extract as much as possible: just before the procedure, a chloroform: methanol solution ( $\geq 99.8 \%$, LiChrosolv ${ }^{\circ}$, Merck, Germany / 24216-2-2.5L-R, 99-99.4\%, 1\% ethanol, Sigma-Aldrich, Germany) was prepared in a 2:1 (v/v) ratio. Of this solution, $40 \mathrm{ml}$ was added to each of the $50 \mathrm{ml}$ tubes containing 4 $\mathrm{g}$ eggs. The tubes were gently shaken for 1 minute and put on hold for 1 minute, and then vortexed for 1 minute. The vortexed suspension was separated from the eggs by filtration, and the filtrate was centrifuged at 1,000 rpm for 10 minutes. Subsequently, the supernatant transferred to a new tube was lyophilized, and the lyophilisate was kept at $-20^{\circ} \mathrm{C}$ until antimycotic susceptibility assay.

\subsection{Determination of antifungal activity}

In order to prepare Roswell Park Memorial Institute-1640 (RPMI-1640) medium, 10.4 g RPMI-1640 (with L-glutamine, without sodium bicarbonate, Sigma, USA) and 0.165 M 34.53 g MOPS (3-N-morpholinopropanesulfonic acid, Sigma, USA) were dissolved in $\mathrm{dH}_{2} \mathrm{O}$. 18g glucose was weighed and added with a glucose concentration of $2 \%$. The $\mathrm{pH}$ was adjusted to 6.9-7.0 with $1 \mathrm{~N} \mathrm{NaOH}$. After the final volume was completed 
to $1,000 \mathrm{ml}$, it was sterilized under aseptic conditions by filtering with a $0.22 \mu \mathrm{m}$ pore-diameter filter (Millipore). The prepared medium was used in experiments after keeping overnight in a $37^{\circ} \mathrm{C}$ and the sterilization controls were performed.

In antifungal susceptibility test, Candida albicans ATCC 10231, Candida parapsilosis ATCC 22019, and Candida tropicalis ATCC 750 standard strains were used. For the preparation of inoculums, 24-hour fresh cultures of all isolates in the Sabauroud Dextrose Agar (SDA) medium were used in the preparation of yeast suspensions. Five colonies with a $\geq 1 \mathrm{~mm}$ diameter size were taken and suspended in $5 \mathrm{ml}$ of $0.85 \%$ sterile saline. The yeast suspensions were homogenized by vortexing them for 15 seconds and then adjusted to 0.5 McFarland turbidity. With this process, stock yeasts containing $1-5 \times 10^{6}$ cells per mililiter were obtained. Stock yeast suspensions were diluted with RPMI1640 medium first at the rate of $1 / 50$ and later at $1 / 20$, and $1-5 \times 10^{3} \mathrm{cell} / \mathrm{ml}$ concentration to be used in the study was reached. The appropriate dilutions were performed using these suspensions and planted in SDA, and the correct concentration was determined by counting the colonies formed at the next day (CLSI M27-A3) (34).

In order to prepare antifungal stock solutions, the tubes containing two-fold drug concentrations between 32-0.06 $\mu \mathrm{g} / \mathrm{ml}$ for amphotericin B were prepared using a pre-prepared antifungal stock suspension and diluted to the degree that was twice as much as the final antifungal concentration. To prepare the egg wax solutions, stock solutions prepared from the waxes dissolved in $10 \mathrm{ml}$ Dimethyl sulfoxide (DMSO) arranged at a concentration of $10,000 \mu \mathrm{g} / \mathrm{ml}$.

In the study, the determination process of the minimal inhibitory concentration (MIC) value was performed as follows: In accordance with antifungal susceptibility test CLSI M27-A3 standards (34), the micro dilution method was employed. Amphotericin B $(32-0.06 \mu \mathrm{g} / \mathrm{ml})$ was used as a control group, and DMSO was used as the negative control in the study. Serial dilutions $(5,000-9.76 \mu \mathrm{g} / \mathrm{ml})$ of the wax stock solutions were performed. The first well of the microplate in the horizontal row was determined as the sterility control, and the last well (12th well) as the reproduction control well. 100 $\mu \mathrm{l}$ RPM-1640 and $50 \mu \mathrm{l}$ RPMI-1640 was added to the sterility well and reproduction control well, respectively. $50 \mu$ of the dilutions were distributed to each column of the microplate as one waxy cover and drug concentration per column. It was dispensed with a pipette, keeping the highest concentration in the second well and the lowest concentration in the $11^{\text {th }}$ well. After the preparation of the microplates, $50 \mu$ l of yeast suspensions arranged as one isolate per row was added. Yeast suspension was not added into the sterility control well. At the end of this process, both yeast and drug concentrations were diluted by $1 / 2$, and study concentrations were reached. After the microplates were covered and incubated at $35^{\circ} \mathrm{C}$ for 48 hours, the susceptibility results were visually evaluated. The lowest concentration without reproduction after incubation was determined as MIC (CLSI M27-A3) (34). The procedures were repeated 3 times using waxy cover obtained from different egg groups of the same species.

\section{RESULTS}

In this study, it was seen that the amount of egg batches obtained from 10 fully satiated females of each tick species would be sufficient to determine the repeated MIC value to be tested against at least three agents. Due to the solvents benefited, it was understood that the use of glass material was ideal at almost all steps of the study. One of the biggest problems that may be encountered in the process is the calculation of the amount of obtained lyophilisate, and at this point, it may be more accurate to try to determine the weight of the waxy cover, which is resistant to repeated dissolution, by dissolving it in a certain amount of solvent after lyophilization. At least, with such an approach, it was seen that it is necessary to confirm the calculations made.

In the trials, no antifungal effect was observed in any of the serial waxy cover concentrations $(5.000-9.76 \mu \mathrm{g} / \mathrm{ml})$ belonging to $H$. marginatum and $D$. marginatus. This result did not change in repetitions. On the other hand, in the serial waxy cover concentrations $(5.000-9.76 \mu \mathrm{g} / \mathrm{ml})$ belonging to $R$. bursa, an inhibition against $C$. tropicalis was identified, the MIC value was found to be $625 \mu \mathrm{g} / \mathrm{ml}$, and the same results were obtained in repeated trials (Table 1 ).

Table 1. Minimum inhibitory concentration (MIC) values of the tick egg waxes and reference substance, Amphotericin B $(\mu \mathrm{g} / \mathrm{ml})$.

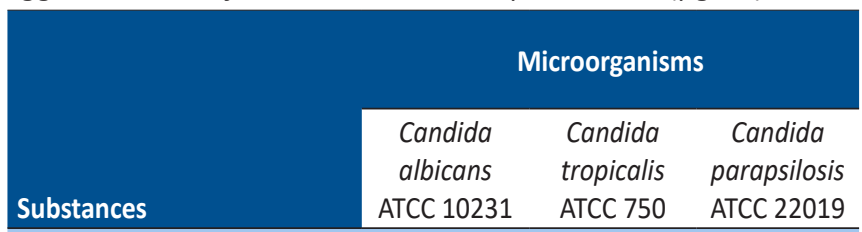

Hyalomma marginatum

Dermacentor marginatus

$\begin{aligned} & \text { Rhipicephalus bursa } \\ & \text { Amphotericin B }\end{aligned}$
$\begin{aligned} & \text { (-) No antimycotic effects were observed at the concentrations of } \leq 5000 \\ & \mu \mathrm{g} / \mathrm{ml} \text {. }\end{aligned}$

\section{DISCUSSION}

Within the life cycle in nature, one of the most critical problems of the ticks is to protect the eggs until the larval hatching and maintain their vitality. The engorged female ticks that detached from the host prefer shady and humid areas such as clefts or cracks on the ground for laying eggs. However, such sites are the places where many pathogens, saprophyte bacteria and fungi are also found. This coexistence 
especially possesses a potential problem for the tick species which have a long period of larval hatching (23). It is known that the egg wax is essential in terms of protecting the eggs from the microbiological, chemical, or physical factors (25, 26). In the studies carried out on some tick species, different type of molecules have been determined in the wax such as long-chain hydrocarbons, branched and unbranched alkanes, fatty acids, cholesterol, and some other steroid types, alcohols $(20,24,26)$, and some specific proteins and lipoproteins $(23,25)$. It was stated that the wax is resistant to some physical and chemical factors such as cold, heat, proteinase $\mathrm{K}$, and pronase (25). However detailed data have not yet been obtained at the point of the determination of the wax content, especially its bioactive substances $(20,26)$.

In the studies carried out to determine the antimicrobial activities of the wax, successful results have been obtained in various degrees against different agents such as Escherichia coli (22, 29), Staphylococcus epidermidis (25-27, 32), Staphylococcus aureus (22, 26, 29), Serratia marcescens (25), Bacillus cereus (26), Bacillus subtilis (25-27), Micrococcus luteus (27, 29, 30), Pseudomonas aeruginosa (22, 31), Enterecoccus faecalis, Enterecoccus faecium, Nocardia asteroides, Salmonella enteritidis, Klebsiella pneumonia (22), picornavirus (28), and influenza virus $(28,29)$. Furthermore, the wax of Boophilus microplus was reported to be effective on C. albicans to some extent (30), and the wax of Rhipicephalus sanguineus was found to be effective on the same agent (29). In our study, no effect was observed related to the wax of $H$. marginatum and $D$. marginatus on examined Candida species. Although similar results were detected from the wax of $R$. bursa against $C$. albicans and C. parapsilosis, some concentration of the wax of this tick species inhibited C. tropicalis (MIC: $625 \mu \mathrm{g} / \mathrm{ml}$ ).

Related studies have obviously shown that the antimicrobial efficacy of tick egg wax is directly correlated to some factors such as the extraction methods and application dose of the wax, and the species of the ticks and microorganisms $(22,25$, 29). The feature of the wax is well known to vary significantly according to the tick species, and it was indicated that this is an expected result, since the sites of egg-laying and habitat preferences of tick species are more or less different from each other $(21,33,35)$. It was also clearly observed in our study, that the differences in the species of ticks and Candida directly affect the results. In accordance with the related information mentioned by the researchers $(20,36)$, our laboratory and field studies conducted in Thrace, Turkey, have revealed that $H$. marginatum and $R$. bursa lay eggs in hot months and in the relatively dry sites and $D$. marginatus during colder months and in more humid area. This difference creates the expectation that the physical and chemical properties of the egg wax of $D$. marginatus can differ from those of other tick species. However, our related studies have shown that the laying and larval hatching procedures of $R$. bursa exhibit significant differences compared to $H$. marginatum and $D$. marginatus. While the whole process of the egg-laying, larval hatching, and larval activation usually take a couple of weeks in the last two species, it can take months in R. bursa. As a result of this delayed process, although the adult stages of this species feed on the host mostly in and around June, the larvae are found on the host in the late autumn or winter (detailed data not presented). In this context, it seems like a natural consequence that the eggs of $R$. bursa are supported by a more featured wax, which ensure adequate protection to the eggs during the relatively long hatching period.

\section{CONCLUSION}

The results obtained from the egg wax of $R$. bursa was interpreted as it could be associated with its some distinctive biological characteristics, and it was concluded that the detailed studies with this and some other tick species that share similar biological and ecological features might yield significant results for the discovery of the new antifungals.

\section{CONFLICT OF INTEREST}

Authors declare no scientific and financial interest.

\section{REFERENCES}

[1] da Silva Dantas A, Lee KK, Raziunaite I, Schaefer K, Wagener J, Yadav B, Gow NAR. Cell biology of Candida albicans-host interactions. Curr Opin Microbiol 2016; 34: 111-118.

[2] Vengurlekar S, Sharma R, Trivedi P. Efficacy of some natural compounds as antifungal agents. Pharmacogn Rev 2012; 6: 9199.

[3] Miceli MH, Díaz JA, Lee SA. Emerging opportunistic yeast infections. Lancet Infect Dis 2011; 11: 142-151.

[4] Papon N, Courdavault V, Clastre M, Bennett RJ. Emerging and emerged pathogenic Candida species: beyond the Candida albicans paradigm. PLoS Pathog 2013; 9: e1003550.

[5] Jahagirdar VL, Davane MS, Aradhye SC, Nagoba BS. Candida species as potential nosocomial pathogens - A review. Electron J Gen Med 2018; 15: em05.

[6] Whaley SG, Berkow EL, Rybak JM, Nishimoto AT, Barker KS and Rogers PD. Azole antifungal resistance in Candida albicans and emerging non-albicans Candida species. Front Microbiol 2017; 7: 2173 .

[7] Wiederhold NP. Antifungal resistance: current trends and future strategies to combat. Infect Drug Resist 2017; 10: 249259.

[8] Jensen RH, Astvad KMT, Silva LV, Sanglard D, Jørgensen R, Nielsen KF, Mathiasen EG, Doroudian G, Perlin DS, Arendrup MC. Stepwise emergence of azole, echinocandin and amphotericin B multidrug resistance in vivo in Candida albicans orchestrated by multiple genetic alterations. J Antimicrob Chemother 2015; 70: 2551-2555.

[9] Wang H, Xu YC, Hsueh PR. Epidemiology of candidemia and antifungal susceptibility in invasive Candida species in the Asia-Pacific region. Future Microbiol 2016; 11:11.

[10] Sarma S, Upadhyay S. Current perspective on emergence, diagnosis and drug resistance in Candida auris. Infect Drug Resist 2017; 10: 155-165.

[11] Shen B. A new golden age of natural products drug discovery. Cell 2015; 163: 1297-1300. 
[12] Abad MJ, Ansuategui M, Bermejo P. Active antifungal substances from natural sources. Arkivoc 2007; 7: 116-145.

[13] Sardi JCO, Scorzoni L, Bernardi T, Fusco-Almeida AM, Mendes Giannini MJS. Candida species: current epidemiology, pathogenicity, biofilm formation, natural antifungal products and new therapeutic options. J Med Microbiol 2013; 62: 1024.

[14] Wang S, Wang Q, Yang E, Yan L, Li T, Zhuang H. Antimicrobial compounds produced by vaginal Lactobacillus crispatus are able to strongly inhibit Candida albicans growth, hyphal formation and regulate virulence-related gene expressions. Front Microbiol 2017; 8: 564.

[15] Wright AE, Bothelo JC, Guzman E, Harmody D, Linley P, McCarthy PJ, Pitts TP, Pomponi SA, Reed JK. Neopeltolide a macrolide from a Lithistid sponge of the family Neopeltidate. J Nat Prod 2007; 70: 412-416.

[16] Park J, Kwon O, An HJ, Park KK. Antifungal effects of bee venom components on Trichophyton rubrum: A novel approach of bee venom study for possible emerging antifungal agent. Ann Dermatol 2018; 30: 202-210.

[17] Führer E Willers D. The anal secretion of the endoparasitic larva Pimpla turionellea: sites of production and effects. J Insect Physiol 1986; 32: 361-367.

[18] Badr AN, Nada F, Shehata MG, Amra HA. Anti-mycotic and anti-mycotoxigenic properties of Egyptian dill. J Applied Sci 2017; 17: 184-195.

[19] Labruna MB, Leite RC, de Oliveira PR. Study of the weight of eggs from six ixodid species from Brazil. Mem Inst Oswaldo Cruz, Rio de Janeiro 1997; 92: 205-207.

[20] Sonenshine DE, Roe RM. Biology of Ticks. Vol. 1, $2^{\text {nd }}$ ed. New York, Oxford University Press; 2014.

[21] Lees AD, Beament JWL. An egg-waxing organ in ticks. Q J Microsc Sci 1948; 89: 291-322.

[22] Yang X, Jia Q, Chen J, Hou Y, Zhai S, Yu Z, Liu J. Antibacterial activity of eggs and egg wax covering of selected Ixodid (Acari: Ixodidae) ticks. J Entomol Sci 2017; 52: 387-394.

[23] Booth TF. Observation on the composition and biosynthesis of egg wax lipids in the cattle tick, Boophilus microplus. Exp Appl Acarol 1992; 14: 137-149.

[24] Matsuo T, Okura N, Kakuda H, Yano Y. Reproduction in a metastriata tick, Haemaphysalis longicornis(Acari: Ixodidae). J Acarol Soc Jpn 2013; 22: 1-23.

[25] Arrieta MC, Leskin BK, Kaufman WR. Antimicrobial activity in the egg wax of the African cattle tick Amblyomma hebraeum (Acari: Ixodidae). Exp Appl Acarol 2006; 39: 297-313.
[26] Yu Z, Thomson ELS, Liu J, Dennis JJ, Jacobs RL, Kaufman WR. Antimicrobial activity in the egg wax of the tick Amblyomma hebraeum (Acari: Ixodidae) is associated with free fatty acids C16:1 and C18:2. Exp Appl Acarol 2012; 58: 453-470.

[27] Lima-Netto $S$, Mendonca RZ, Franzolin $M R$, Utescher $C L$, Orozco S, Máximo-Espindola C, Labruna M, Barros-Battesti DM. An interesting antimicrobial activity of egg wax from Amblyomma cajennense (Acari: Ixodidae). Syst Appl Acarol 2011; 16: 3-6.

[28] Lima-Netto S, Pinheiro A, Nakano E, Mendonca RMZ, BarrosBattesti DM, Mendonca RZ. Antiviral effect of the egg wax of Amblyommacajennense (Acari: Ixodidae). Cytotechnology 2012; 64: 601-606.

[29] Alduini N, Silva M, Franzolin M, Mendonça R, Lima-Netto S. Antimicrobial activity from ticks eggs waxes. BMC Proceedings 2014; 8: 156.

[30] Esteves E, Fogaca AC, Maldonado R, Silva FD, Manso PPA, Pelajo-Machado M, Valle D, Daffre S. Antimicrobial activity in the tick Rhipicephalus (Boophilus) microplus eggs: Cellular localization and temporal expression of microplusin during oogenesis and embryogenesis. Dev Comp Immunol 2009; 33: 913-919.

[31] Zimmer KR, Macedo AJ, Nicastro GG, Baldini RL, Termignoni C. Egg wax from the cattle tick Rhipicephalus (Boophilus) microplus inhibits Pseudomonas aeruginosa biofilm. Ticks and Tick-borne Dis 2013a; 4: 366-376.

[32] Zimmer KR, Macedo AJ, Giordani RB, Conceição JM, Nicastro GG, Boechat AL, Baldini RL, Abraham WR, Termignoni C. A steroidal molecule present in the egg wax of the tick Rhipicephalus (Boophilus) microplus inhibits bacterial biofilms. Environmental Microbiol 2013b; 15: 2008-2018.

[33] Sonenshine DE, Tigner JA. Oviposition and hatching in two species of ticks in relation to moisture deficit. Ann Entomol Soc Am 1969; 62: 628-640.

[34] Clinical and Laboratory Standard Institute (CLSI). Reference method for broth dilution antifungal susceptibility testing of yeasts-third edition: approved standard M27-A3. Wayne: CLSI; 2008.

[35] Teel PD. Effect of Saturation Deficit on Eggs of Boophilus annulatus and B. microplus (Acari: Ixodidae). Ann Entomol Soc Am 1984; 77: 65-68.

[36] Estrada-Peña A, Mihalca AD, Petney TN. Ticks of Europe and North Africa: A Guide to Species Identification. $1^{\text {st }}$ ed. Switzerland, Springer International Publishing; 2017.

How to cite this article: Bilgin N, Hacioglu M, Bozkurt Guzel C, Erdal B, Kar S. In-vitro anticandidial efficacy of tick egg wax from Hyalomma marginatum, Rhipicephalus bursa and Dermacentor marginatus. Clin Exp Health Sci 2020; 10: 108-112. DOI: 10.33808/ clinexphealthsci.707924 